\title{
Loop transfer recovery with minimal-order observers
}

\section{Søgaard-Andersen, Per}

Published in:

26th IEEE Conference on Decision and Control

Link to article, DOI:

10.1109/CDC.1987.272530

Publication date:

1987

Document Version

Publisher's PDF, also known as Version of record

Link back to DTU Orbit

\section{Citation (APA):}

Søgaard-Andersen, P. (1987). Loop transfer recovery with minimal-order observers. In 26th IEEE Conference on Decision and Control (Vol. Volume 26, pp. 933-938). IEEE. https://doi.org/10.1109/CDC.1987.272530

\section{General rights}

Copyright and moral rights for the publications made accessible in the public portal are retained by the authors and/or other copyright owners and it is a condition of accessing publications that users recognise and abide by the legal requirements associated with these rights.

- Users may download and print one copy of any publication from the public portal for the purpose of private study or research.

- You may not further distribute the material or use it for any profit-making activity or commercial gain

- You may freely distribute the URL identifying the publication in the public portal

If you believe that this document breaches copyright please contact us providing details, and we will remove access to the work immediately and investigate your claim. 
LOOP TRANSFER RECOVERY WITH MINIMAL-ORDER OBSERVERS.

Per Søgaard-Andersen

Control Engineering Institute

Technical University of Denmark

Builaing 424, 2800 Lyngby - Denmark

\section{Abstract}

A solution to the problet. of 1000 transfer recovery with minimal order observers is obtained for the control loop broken at the plart input. Three distinct cases are treated - dependirg on the properties of the first Markov parameter of the plant model. For two of these cases a LQG-type desicn method is outlined. If the first Markov parameter is of full rank exact loop transfer recovery can be actieved.

\subsection{Introduction.}

In recent years a number of new tools for the design of robust miltivariable control systems have emerged. Among these is the multivariable singularvalue lcop-shaping paradigm [D1,S2], based on the LQG/ ITR design approach [Dl,Al]. In this context ioop transfers designed to met certain stability robustness and loop performance requirements can be reccvered asymptoticaliy by suitable (filter or full state) designs.

In [SI,Dl,Al] this design approach is derived based or flill-order observers, hence the resulting compensator will be of the same order as the plant. If the plant is of high order this may lead to compensators of very high orders. In [A2] a method for reducing the order of the compensator is outlined. Such methods may, however, reduce the performance of the control loop, or in other cases it is not applicable. As an alternative reduced order observers can be employed.

Dowdie [D2] has shown that it is not generally feasible to use observers of reduced order in LTRschemes, but in the minimal-order case (see [01] for a precise definition) a solution is possible. Such comcensators have been studied in $[\mathrm{D} 2, \mathrm{D} 3, \mathrm{M} 2]$. In $[\mathrm{D} 2, \mathrm{D} 3]$ it is required that the first Markov parameter of the plant model is zero. This restriction is not required in [M2], instead it is required that certain matrices are of full rank and that a certain subsystem of $S(A, B, C)$ is minimum-phase.

Such restrictions are not imposed here, and a solution to the minimal-order ITR problem is derived.

The paper is organized as follows. In $\$ 2$ the loop shape philosophy is briefly outlined, and in $\$ 3$ the notation is presented. In $\S 4$ the analysis is performed and the design issues are treated in $\$ 5$ followed in $\$ 6$ by two examples, and in $\$ 7$ by some summarizing remarks.

\subsection{The 10op-shape design philosophy.}

Assume that the physical plant $G(s)$ differs from the plant model $G_{0}(s)$ in tine following way

$$
\begin{aligned}
& G(s)=G_{0}(s)(I+L(s)) \\
& \bar{\sigma}(L(s)) \leq \hat{i}(j \omega)
\end{aligned}
$$

This representation is known as multiplicative modelling errors at the plant input. If $C$ (s) is a compensator the closed-loop system is stable for $t(s)$ if [D1]

$$
\underline{\sigma}\left(I+\left(C G_{0}\right)^{-1}\right)>\hat{\lambda}(j \omega) \quad \omega>0
$$

Further let the performance objectives be expressed as $[D I, S 1, M 3]$

$$
\underline{\sigma}\left(I+C G_{0}\right)>Y(j \omega) \quad \omega \geq 0
$$

Notice that the performance objectives should be reflected to the same plant node as the uncertainties [S1, v3]. These two constrairts specify frequency-dependent bounds on the loop transfer $C_{C}$. If the constraints are not contradictory a full state Eeedback. design which satisfies these bounds can be achieved by a suitable $L$ weight selection [AI,DI]. The full state loop transfer can be recovered asymptotically by an LTR observer design, and consequently the resulting model-based compensator has the same loop properties. A dual procedure for the plant output node can also be outlined.

A detailed practical example of this procedure has been considered in [A2].

Hence robust stability and nominel performance can be satisfied. The issue of robust performance can also be formulated in the loop shape setting for some problems. Details of this is discussed in [S1,M3].

In the following it is assumed that a full state feedback has been derived such that the full state loop transfer satisfies the design inequalities (2-2, 2-3). Next it is desired to recover this (target) feedback loop with a minimal order observer.

\subsection{Minimal-order observers.}

Let the FDLTI plant model be represented by a minimal state-space realization:

$$
\begin{aligned}
& \dot{x}=A x+B u \quad x \in R^{n} \quad u \in R^{2} \\
& y=C x \quad y \in R^{m}
\end{aligned}
$$

with $m=r, n>m$ and $C, B$ of full rank. Further let $s(A$, $B, C)$ be minimum-phase. The control signal is given by

$$
u=-\hat{k x}+r
$$

where $\mathrm{x}$ is the state estimate.

In the following the notation of minimal order observers is briefly introduced. This notation is similar to the notation in the monograph by O'Reilly [0I]. 
The structure of the observer is shown in figure 1 . The dimensions of the signals are indicated in the brackets.

Let $S(A, B, C)$ be partitioned as:

$$
\begin{aligned}
& A=\left[\begin{array}{cc}
A_{11} & A_{12} \\
A_{21} & A_{22}
\end{array}\right] \uparrow_{n-m}^{m} \quad B=\left[\begin{array}{c}
B_{1} \\
B_{2}
\end{array}\right] \uparrow_{n-m}^{m} \quad C=\underbrace{\left[\begin{array}{ll}
I_{m} & 0
\end{array}\right]}_{n-m} \\
& \text { m. } \mathrm{n}-\mathrm{m}
\end{aligned}
$$

A similarity transformation can always be selected to bring $S(A, B, C)$ in this form.

With this partitioning - and without loss of generality - the minimal order observer matrices are [01].

$$
\begin{aligned}
& G=B_{2}-V_{2} B_{1}, \quad D=A_{22}-V_{2} A_{12} \\
& E=A_{21}-V_{2} A_{11}+A_{22} V_{2}-V_{2} A_{12} V_{2} \\
& P=\left[\begin{array}{c}
0 \\
I_{n-m}
\end{array}\right], \quad V=\left[\begin{array}{c}
I_{m} \\
V_{2}
\end{array}\right]
\end{aligned}
$$

O'Reilly refers to this class of minimal order observers as a "parametric class" of observers, since the observers are completely specified by the arbitrary gain matrix $v_{2}$. This matrix takes the place of the observer gain in minimal-order design.

The loop transfer for the plant input loop-breaking point is:

$$
\begin{aligned}
& I_{x}(s)=K\left(I+P{ }_{22}^{\prime} G K\right)^{-1}\left(V+P \Phi_{22}^{\prime} E\right) G_{0} \\
& S_{22}^{\prime}(s)=\left(s I-A_{22}+V_{2} A_{12}\right)^{-1} \\
& G_{0}(s)=C \Phi(s) B
\end{aligned}
$$

For this feedback system the separation principle applies. A detailed derivation of this result can be found in [01]. This implies that the full-state and the observer design can be carried out separately.

Arbitrary pole-placement of the error dynamics requires $(C, A)$ to be observable. If $(C, A)$ is an observable pair, this implies that $\left(\mathrm{A}_{12}, \mathrm{~A}_{22}\right)$ is observable [01]. It is therefore assumed $\operatorname{that}^{22}$ (C,A) is observable.

\subsection{ITR with minimal order observers}

The condition for ITR for the minimal order observerbased feedback system is that the loop transfer $L_{x}(s)$ equals the full state loop transfer $K \Phi(s) B$. The derivation of the recovery condition is lengthy but straightforward. As a consequence the derivation is omitted here. The condition is [M2]:

$$
v_{2}\left(I+A_{12} \Phi_{22}(j \omega) v_{2}\right)^{-1} A_{12} \Phi_{22}(j \omega)\left(B_{2}-v_{2} B_{1}\right)=B_{2}-V_{2} B_{1},
$$

$\forall \omega$

$$
\Phi_{22}(s)=\left(s I-A_{22}\right)^{-1}
$$

A number of straightforward manipulations can bring eq. $(4-1)$ in the form:

$$
V_{2}\left(I+A_{12} \Phi_{22} V_{2}\right)^{-1}=B_{2}\left(B_{1}+A_{12} \Phi_{22} B_{2}\right)^{-1}
$$

This is the necessary and sufficient condition for LTR with minimal order observers, and the equivalent to the full-order condition. Unfortunately the condition is not as simple, and the design implications on $\mathrm{v}_{2}$ are more involved.

To be more specific it turns out that eq. (4-2) implies 3 different design cases depending om the rank of $B_{1}$. The details of the analysis for the three cases are treated in appendix $A$

Case $1-\operatorname{rank}\left(B_{1}\right)=0$ - In this case the recovery conäition is:

$$
\mathrm{V}_{2}\left(\mathrm{I}+\mathrm{A}_{12} \Phi_{22} \mathrm{~V}_{2}\right)^{-1}=\mathrm{B}_{2}\left(\mathrm{~A}_{12} \Phi_{22} \mathrm{~B}_{2}\right)^{-1}
$$

which is similar to the full order condition. Therefore the gain $v_{2}$ must be selected so that:

$$
\frac{v_{2}(q)}{q} \rightarrow B_{2} \alpha, q \rightarrow \infty
$$

For some nonsingular $\alpha$.

As $q$ increases the poles of $\mathrm{A}_{22}-\mathrm{V}_{2} \mathrm{~A}_{12}$, which grovern the error dynamics, will behave in the following way:

i) $p$ poles move towards the zeros of $S(A, B, C)$.

ii) $n-m-p$ poles move towards infinity in $m$ Butterworth patterns of orders deter-mined by the projected Markov parameters [KI] of $S\left(A_{22}, B_{2}, A_{12}\right)$, or certain Toeplitz matrices [V1].

Since rank $\left(B_{1}\right)=\operatorname{rank}(C B)$ such systems can maximally have $n-2 m$ zeros [MI].

Case 2 - rank $\left(B_{1}\right)=m$ - The recovery condition is after reordering:

$$
\begin{gathered}
\mathrm{V}_{2}\left(\mathrm{I}+\mathrm{A}_{12} \Phi_{22} \mathrm{~V}_{2}\right)^{-1}=\mathrm{B}_{2} \mathrm{~B}_{1}^{-1}\left(\mathrm{I}+\mathrm{A}_{12} \Phi_{22} \mathrm{~B}_{2} \mathrm{~B}_{1}^{-1}\right)^{-1} \\
\mathrm{v}_{2}=\mathrm{B}_{2} \mathrm{~B}_{1}^{-1}
\end{gathered}
$$

and $v_{2}$ is uniquely determined.

The condition is $\operatorname{rank}\left(B_{1}\right)=\operatorname{rank}(C B)=m$, i.e. the first Markov parameter is of full rank. Therefore $S(A, B, C)$ has $n-m$ zeros [MI]. With $V_{2}$ as in eq. (4-5) the eigenvalues of $A_{22}-V_{2} A_{12}$ are equal to the zeros of $S(A, B, C)$.

In this special case it is therefore possible to achieve perfect recovery for an observer-gain matrix with finite gains.

Case $3-0<\operatorname{rank}\left(B_{1}\right)<m$ - The recovery condition now implies:

$$
\frac{\mathrm{v}_{2}(\mathrm{q})-\mathrm{B}_{2}{ }^{\beta}}{\mathrm{q}} \rightarrow \mathrm{B}_{2}{ }^{\alpha} \quad, \quad \mathrm{q} \rightarrow \infty
$$


$a \in \operatorname{Ker}\left(B_{1}\right)$

and $B$ must be selected as $\mathrm{B}_{1}^{+}$, then eq. $(4-6)$ results in

i) poles nove towards the zeros of $S(A, B, C)$.

ii) $n-m-p$ poles move towaràs infinity in m-rank $\left(B_{1}\right)$ Butzerworth patterns.

In the following it is assumed that:

$$
B_{1}=\left[\begin{array}{ll}
1 & 0 \\
0 & 0
\end{array}\right]
$$

which can always be obtained with a suitable transformation. Now eq. $(4-6)$ becoms:

$$
\begin{aligned}
& \frac{v_{2}(q)-B_{2} B_{1}^{+}}{q} \rightarrow B_{2}\left(\begin{array}{ll}
0 & 0 \\
0 & \alpha
\end{array}\right) \\
& v_{2}(q)=B_{2} B_{1}^{+}+v_{2 q} \\
& \frac{v_{2 q}}{q} \rightarrow B_{2}\left(\begin{array}{ll}
0 & 0 \\
0 & \alpha
\end{array}\right)
\end{aligned}
$$

Let $A_{12}, B_{2}$ and $v_{2 q}$ be partitioned as:

$$
\begin{aligned}
& A_{12}=\left.\left[\begin{array}{l}
A_{121} \\
A_{122}
\end{array}\right]\right|_{i} \begin{array}{l}
r\left(B_{1}\right) \\
m-r\left(B_{1}\right)
\end{array} \\
& B_{2}=\left[\begin{array}{ll}
B_{21} & B_{22}
\end{array}\right] \uparrow n-m \\
& \overrightarrow{r\left(B_{1}\right) m-r\left(B_{1}\right)}
\end{aligned}
$$

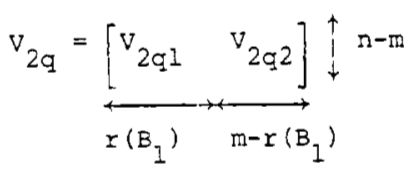

It is then found that:

$$
v_{2 q l}=0, \frac{v_{2 q 2}}{q} \rightarrow E_{22^{a}}
$$

is the recovery condition. If $v_{2}$ is chosen as in eq. $(4-8)$ the following applies?

i) p poles move towards the zeros of $S(A, B, C)$.

ii) n-m-p poles move towards infinity in m-rank $\left(B_{1}\right)$ Butterworth patterns of orders determined by the projected Markov paramters of $\mathrm{S}\left(\overline{\mathrm{A}}_{22}, \mathrm{~B}_{22}, \mathrm{~A}_{122}\right), \overline{\mathrm{A}}_{22}=\mathrm{A}_{22}-\mathrm{B}_{21} \Lambda^{-\mathrm{I}_{\mathrm{A}}}{ }_{121}$ or certain Toeplitz matrices.

If $\operatorname{rank}\left(B_{1}\right)=d$ such systems can maximally have $n-$ $2 \mathrm{~m}+\mathrm{d}$ zeros $[\mathrm{M} 1]$.

If $B_{1}$ is not diagonal a similarity transformation is needea. The corresponding expressions are:

$$
B_{1}=T\left[\begin{array}{ll}
\Lambda & 0 \\
0 & 0
\end{array}\right] T^{-1}, \quad B_{1}^{\prime}=\left[\begin{array}{ll}
\Lambda & 0 \\
0 & 0
\end{array}\right]
$$

$$
\begin{aligned}
& A_{12}^{\prime}=T^{-1} A_{12}, B_{2}^{\prime}=B_{2} T, V_{2}=\left(V_{20}^{\prime}+V_{2 q^{\prime}}\right) T^{-1} \\
& V_{20}^{\prime}=B_{21}^{\prime} \Lambda^{-1}, \frac{V_{2 q}^{\prime}}{q} \rightarrow B_{22}^{\prime} \alpha^{\prime}
\end{aligned}
$$

Here the prime indicates the new coordinate frame. If $\mathrm{B}_{1}$ is not diagonalizable the recovery conditions still apply but the asymptotic behaviour is more involved. Notice that the first and second case be considered as the limits of the third case as rank $\left(B_{1}\right)$ is either 0 or $\mathrm{m}$.

An important effect of minimal order $I T R$ is that $\max n-m-p$ poles approach infinity (in the limit). In the fullorder case $n-p$ poles approach infinity. clearly the number of infinite zeros is reduced. The covery conditions are similar to the full order observer case. The similarity is emphasized in table 1.

The term "subject to" indicates that the recovery condition is imposed on a feedback loop with the elements $S(x, x, x)$. The similarity will be useful in finding simple design rules.

In Case $2 \mathrm{~V}_{2}$ is uniquely given, so design will only be considered tor the 2 other cases.

\subsection{LQG-methods in minimal order LTR}

The recovery conditions found in $\S 4$ form the basis of the ITR-procedures for minimal observers.

Based on the "parametric class" of minimal order observers ( $\$ 3$ ) optimality conditions similar to the full-order LQG-conditions can be derived (e.g. see Dowdle [D 2]). However, optimality in some mathematical sense is not of prime interest in this context. Here the focus is on design methods that are relevant in achieving loop transfer recovery. Hence optimality in the strict mathematical sense will not be pursued.

In the following two subsections such recovery methods will be considered for case 1 and case 3 . The methods are based on suitably chosen Riccati-equations.

\section{Minimal order LQG/LTR - Case 1}

The recovery condition is given by:

$$
\frac{V_{2}(q)}{q} \rightarrow B_{2} \alpha \quad \text { subject to } S\left(A_{22}, B_{2}, A_{12}\right)
$$

Now consider the filter Riccati-equation:

$$
\begin{aligned}
& \mathrm{A}_{22} \mathrm{~S}+\mathrm{SA}_{22}{ }^{\mathrm{T}}+\Gamma-\mathrm{SA}_{12}{ }^{\mathrm{T}} \Sigma^{-1} \mathrm{~A}_{12} \mathrm{~S}=0 \\
& \mathrm{v}_{2}=\mathrm{SA}_{12}{ }^{\mathrm{T}} \mathrm{T} \quad, \quad \Sigma>0
\end{aligned}
$$

with the weights selected as:

$$
\Gamma=\Gamma_{0}+q^{2} B_{2} v_{2}{ }^{T}>0, v>0, q \rightarrow \infty
$$

If $S\left(A_{22}, B_{2}, A_{12}\right)$ is minimum-phase $\left(A_{12}, A\right)$ is observable and $\left(A_{22}, I^{\frac{1}{2}}\right)$ is stabilizable the solution to eq. (5-2) asymptotically $(q \rightarrow \infty)$ behaves as:

$$
\frac{V_{2}}{q} \rightarrow B_{2} V^{\frac{1}{2}} \theta \Sigma^{-\frac{1}{2}}
$$

With $\theta$ as some orthonormal matrix. Clearly the recovery condition is met. Further the Riccati-equation 
implies that the eigenvalues of $A_{22}-V_{2} A_{12}$ (i.e. the minimal-order observers dynamics) are stable for any q., if $\left(A_{22}{ }^{T}, A_{12}{ }^{T}, \Gamma^{\frac{1}{2}}\right)$ is minimal. Hence - due to the minimal-order observer separation principle - the overall closed-loop system will be stable and recovery is achieved simultaneously.

The only serious restriction imposed here is that $S\left(A_{22}, B_{2}, A_{12}\right)$ must be minimum-phase. However, the zeros of $S\left(A_{22}, B_{2}, A_{12}\right)$ are equal to the zeros of $S(A, B$, C) [s3]. Hence no new constraints are imposed on the original system.

\section{Minimal-order LQG/LTR - Case 3}

In this case the recovery condition is:

$$
\begin{aligned}
& \frac{\mathrm{V}_{2 q 2}}{q} \rightarrow B_{22} \text { subject to } s\left(\bar{A}_{22}, B_{22}, A_{122}\right) \\
& v_{2}=\left[B_{21} \Lambda^{-1}, v_{2 q 2}\right] T^{-1}
\end{aligned}
$$

To achieve this condition consider the Riccatiequation:

$$
\begin{aligned}
& \overline{\mathrm{A}}_{22} \mathrm{~S}+\mathrm{SA} \overline{\mathrm{A}}_{22}+\Gamma-\mathrm{SA}_{122} \mathrm{~T}_{\Sigma}{ }^{-1} \mathrm{~A}_{122} \mathrm{~S}=0 \\
& \mathrm{~V}_{2 \mathrm{q} 2}=\mathrm{SA}_{122} \mathrm{~T}_{\Sigma}, \quad \Sigma>0
\end{aligned}
$$

with the weight-selection:

$$
\Gamma=\Gamma_{0}+q^{2} B_{22} v_{22}^{T}>0, v>0, q \rightarrow \infty
$$

Constrained only by $S\left(\bar{A}_{22}, B_{22}, A_{122}\right)$ being minimumphase, $\left(\bar{A}_{22}, \Gamma^{\frac{1}{2}}\right)$ stabilizable and $\left(A_{122}, \bar{A}_{22}\right)$ being observable.

As $q$ approach infinity the solution to eq. (5-6) behaves as:

$$
\frac{v_{2 q 2}}{q}+B_{22} v^{\frac{1}{2}} \theta \Sigma^{-\frac{1}{2}}
$$

which shows that the recovery condition is satisfied with observer dynamics given by:

$$
\begin{aligned}
& \lambda_{i}\left[A_{22}-V_{2} A_{12}\right]=\lambda_{i}\left[A_{22}-\left[B_{21} \Lambda^{-1}, v_{2 q 2}\right]-\right. \\
& {\left[\begin{array}{l}
A_{121} \\
A_{122}
\end{array}\right]=\lambda_{i}\left[\bar{A}_{22}-V_{2 q 2} A_{122}\right]}
\end{aligned}
$$

Due to the Ricoatti-equation all observer eigenvalues are stable for any $q$. Hence the overall system is stable. The minimum-phase constraint does not impose any new restrictions on $S(A, B, C)$ since the zeros of $S(A, B, C)$ are equal to the zeros of $S\left(\bar{A}_{22}, B_{22}\right.$ ' $\left.A_{122}\right)$.

Apart from the nice properties of Riccati-equation based methods, these results provide computationally simple approaches to LTR-designs.

\subsection{Examples.}

Two simple examples are considered to illustrate case 2 and 3 .

Case $I$ is illustrated in [D3].

Example 1

Let

$$
\begin{aligned}
& A=\left[\begin{array}{cc}
-2 & 1 \\
0 & -3
\end{array}\right] \quad B=\left[\begin{array}{c}
1 \\
-2
\end{array}\right] \quad C^{T}=\left[\begin{array}{l}
1 \\
0
\end{array}\right] \\
& G(s)=\frac{(s+1)}{(s+2)(s+3)} \\
& K=\left(\begin{array}{ll}
-6 & -5
\end{array}\right)
\end{aligned}
$$

This selection of $\mathrm{K}$ imply that the full state loop transfer is

$$
L(s)=\frac{4 s+14}{(s+2)(s+3)}
$$

The loop transfer with the minimal-order observer in the loop is - after some algebra:

$$
L_{1}(s)=-\frac{s\left(6+5 V_{2}\right)+\left(25 v^{2}+96 V_{2}+78\right)}{s+13+6 V_{2}} \cdot \frac{(s+1)}{(s+2)(s+3)}
$$

with $\mathrm{V}_{2}=\mathrm{B}_{2} \mathrm{~B}_{1}^{-1}=-2$ inserted thes reduces to $\mathrm{L}(\mathrm{s})$ and LTR is achieved exactly - as expected.

Example $\underline{\text { E. }}$

Let

$$
\begin{aligned}
& A=\left[\begin{array}{rrrrrrr}
2 & 0 & 1 & 0 & 0 & 1 & 1 \\
-1 & 2 & 0 & 0 & 0 & 0 & 0 \\
3 & 2 & -1 & 0 & 0 & -2 & 1 \\
2 & -2 & 0 & -4 & 2 & 0 & -1 \\
0 & 2 & 3 & 0 & -2 & 1 & -1 \\
1 & 0 & 2 & -3 & 2 & 2 & 0 \\
-1 & -1 & 1 & 0 & 0 & -1 & 1
\end{array}\right] \quad B=\left[\begin{array}{rrr}
2 & 0 & 0 \\
1 & 0 & 0 \\
1 & 0 & 0 \\
0 & 2 & 0 \\
0 & 3 & 0 \\
0 & 0 & 1 \\
0 & -1 & 0
\end{array}\right] \\
& C=\left[\begin{array}{ll}
I_{3} & 0 \\
3 \times 4
\end{array}\right]
\end{aligned}
$$

$S(A, B, C)$ has two transmission zeros at $(-2,-4)$. The full state feedback is an IQ-design with weights $Q=I_{7}$ and $\mathrm{R}=10^{-3} \mathrm{I}_{3}$.

Since $\operatorname{rank}(C B)=1$, this example belongs to case 3 . Now $v_{2}(q)$ is determined by:

$$
\begin{aligned}
& V_{2}=V_{2}^{\prime} \cdot T^{-1}, V_{2}^{\prime}=\left[B_{21}^{\prime} \cdot \Lambda^{-1} V_{2 q} 2^{\prime}\right] \\
& T=\left[\begin{array}{ccc}
1 & 0 & 0 \\
1 / 2 & 1 & 0 \\
1 / 2 & 0 & 1
\end{array}\right], \bar{A}_{22}=\left[\begin{array}{cccc}
-4 & 2 & -1 / 2 & -3 / 2 \\
0 & -2 & 1 / 4 & -7 / 4 \\
-3 & 2 & 7 / 4 & -1 / 4 \\
0 & 0 & -1 & 1
\end{array}\right]
\end{aligned}
$$

$\mathrm{V}_{2 q 2}$ is determined by the Riccati-equation (5-6). In figure 4 singular value plots of the full state loop transfer and the minimal-order observer loop transfer is shown for different q-values. For $q$ even larger better recovery can be achieved.

\subsection{Summarizing remarks.}

The miminal-order observers are of order $n-m$, whereas full-order observers are of order $\mathrm{n}$. Since the 
LTR synthesis imply that $p$ poles move towards the plant zeros, respectively $n-m-p$ and $n-p$ observer poles must be moved towards infinity. Clearly the number of asymptotically fast modes are reduced by $m$ when minimal-order observers are appiled. This fact enhances the applicability of the minimal-order ITR concept. Further this fact imply that if the number of plant zeros is $n-m$ asymptotically fast modes are not needed in the observer, and exact recovery can be achieved. If $\mathrm{p}<\mathrm{n}-\mathrm{m}$ only asymptotic recovery is possible and two cases emerge. For rank $(C B)=0$ the results given here are equivalent to those in $[D 2, D 3]$.

In the last case the resulting observer gain will contain a high gain and a low gain part. In the limit this may cause numerical problems. Therefore - for practical problems - the q-values are limited. Notice that this case requires the number of irputs to be larger than or equal to 2 , hence this is a multivariable pheromenon.

In this paper a LQG-type of synthesis is proposed. In [53] it is shown that eigenspace methods are also applicable.

The loop-shape formulation used here require that the uncertainties and performance specifications are reflected to the plant imput. Unfortunately similar results for the plant output can not be derived since the minimal-order observer and the plant model are not dual. The results are therefore limited to asymptotic filter designs.

For non-minimum-phase plants the synthesis results still applies, but ITR is not quaranteed over the enire irequency-range (see [s2] for more details on this issie.).

Finally notice that the loop-shape robustness formulation are only well-suited for certain classes of problems, as discussed in $[51, \mathrm{M} 3]$. In more involved robust design problems more refined tools - like the structured singular value [D4] - are required.

\section{References.}

[A1] Athans,M. - A tutorial on the LQG/ITR method, Proc. American Control Conf., Seattle, wa,p. 128996, june $: 986$.

[A2] Athans, M. et.al. - Linear Luadratic Gaussian with loop transfer recovery for the F-100 engine, J. Guidance, 9,1,p. 45-52, 1986

[DI] Doyle, J.C. \& Stein,G. - Multivariable feedback design: Concepts for a classical/modern synthesis, IEEE Transact. Aut Control, AC-26,1,p.4-16, 1981.

[D2] Dowdle, J.R. et.al. - Minimal order robust observer-based compensator, IEEE Conf. Dec. and Control, orlando,FL,F. 894-6, 1982.

[D3] Dowale,J.R. - Robust observer basea compensators, Ph.D. thesis, MIT, Cambridge, MA, USA, 1979.

[D4] Doyie, J.C. - Structured uncertainty in control system design, IEEE Conf. Dec. Control,ft. Lauterdale, Ė, P. 260-5, 1985.

[KI] Kouvaritakis,B. \& Edrunds, J.M. - Multivariable root loci: A unified approach to finite and infinite zeros, Int. J. Control,29,3,p. 393-428, 1979.

[M1] Macfarlane, A.G.J. \& Karcanias, N. - Poles and zeros of linear multivariable systems: A survey of the algebraic, geometric and complex-variable theory, Int. J. Control, 24,1,2.33-74, 1976.

[M2] Madiwale,A.N. \& Williams,D.E. - Some extensions of loop transfer reccuery, Proc. American Control Conf., Boston, MA, p. 790-5, 1985.

[M3] Morari,M. \& Doyle, J.C. - A unifying framework for control system design under uncertainty and its implications for chemical proces control,
Third Intl. Conf, on Chem. Proc. Control, Asilomar, $\mathrm{Ca}, \mathrm{p} .5-51,1986$.

[OI] O'Reilly,J. - Observers for linear systems, Academic Press, London 1983.

[S1] Stein,G. - Beyond singular values and loop shapes, Rep.no. IIDS-P-1504 IIDS, MIT, MA, USA, 1985.

[S2] Stein,G. \& Athans M. - The LQG/LTR design procedure for multivariable feedback design, Rep. no LIDS-p-1384, LIDS, MIT, MA, 1984.

[s3] Søgaard-Andersen,P. - Issues in robust multivariable observer-based feedback design. Ph.D. thesis, Control Engng. Inst., Tech. Univ. of Denmark, Lyngby, 1987.

[vi] Verghese,G. \& Kailath,T. - Comments on "On structure invariants and the root-loci of linear multivariable stytems, Int.J. Control,29,6, p. $1077-80,1979$.

APPENDIX A.

Analysis of the recovery condition for minimal order observers.

The general condition for LTR with minimal order observers is:

$$
V_{2}\left(I+A_{12} \frac{\Phi}{\omega} 22(j \omega) V_{2}\right)^{-1}=B_{2}\left(B_{1}+A_{12} \delta_{22}(j \omega) B_{2}\right)^{-1}, \forall \omega
$$

The aralysis of the condition is divided into three cases.

Cace $1 r\left(B_{-}\right)=0$ - By inspection the resuiting LTR condition is equivalent to the full-order recovery condition. Hence the gain $v_{2}$ must be selected so that:

$$
\frac{V_{2}(q)}{q}+B_{2} \alpha \quad, \quad q \rightarrow \infty
$$

The eigenvalues of $\mathrm{A}_{22}-\mathrm{V}_{2} \mathrm{~A}_{12}$ as $\mathrm{q} \rightarrow \infty$ are the roots of the closed-loop characteristic polynomium (CLCP)

$$
I+A_{12} \Phi_{22} V_{2}^{\prime}=\frac{C L C P}{O L C P}, \quad ! !=\operatorname{det}(\cdot)
$$

with OLCP as the open-loop characteristic polynomium.

By combining $(A-3)$ and The schur determinant formula:

$$
\frac{1}{\mathrm{q}} \mathrm{CLCP} \rightarrow\left|\begin{array}{cc}
\mathrm{a}^{-1} & \mathrm{~B}_{2} \mathrm{a}^{\prime} \\
-\mathrm{A}_{12} & \mathrm{I} / \mathrm{q}
\end{array} \rightarrow\right| \begin{array}{cc}
\text { sI-A } & \mathrm{B} \alpha \\
-\mathrm{C} & 0
\end{array} \mid
$$

where the last convergence follows from the structure of $C$.

It is thus clear that CLCP $=0$ if the closed-loop eigenvalues approach the $p$ zeros of $S(A, B, C)$ as $q \rightarrow \infty$, since $B_{1}=0$. The remaining eigenvalues go to infinity in $\mathrm{m}$ Butterworth patterns. The results of [s3] imply that the order of approach is determined by the projected Markov parameters of $\mathrm{S}\left(\mathrm{A}_{22}, \mathrm{~B}_{2}, \mathrm{~A}_{12}\right)$ [KI].

Case $\& r\left(B_{1}\right)=m$ - The condition is now: 


$$
V_{2}\left(I+A_{12} \Phi_{22} V_{2}\right)^{-1}=B_{2} B_{1}^{-1}\left(I+A_{12} \Phi_{22} B_{2} B_{1}^{-1}\right)^{-1}
$$

and $V_{2}=B_{2} B_{1}^{-1} . V_{2}$ is thus uniquely determined by $B$. The resulting eigenvalues of $\mathrm{A}_{22}-\mathrm{V}_{2} \mathrm{~A}_{12}$ are the roots of:

$$
\begin{aligned}
\text { CICP } & =\left|\Phi_{22}^{-1}\right|\left|I+A_{12} \Phi_{22} B_{2} B_{1}^{-1}\right| \\
& =\left|\Phi_{22}^{-1}\right|\left|B_{1}+A_{12} \Phi_{22} B_{2}\right| B_{1} \mid \\
& =\left|\begin{array}{ll}
\Phi_{22}^{-1} & B_{2} \\
-A_{12} & B_{1}
\end{array}\right|\left|B_{1}\right|=\left|\begin{array}{ll}
\text { I-A } & B \\
-C & 0
\end{array}\right| B_{1} \mid
\end{aligned}
$$

Clearly the roots of CLCP are the zeros of $S(A, B, C)$. Since rank $(C B)=m S(A, B, C)$ has $n-m$ zeros $[M I]$. AII the $n-m$ roots of CLCP are thus equal to a zero of $S(A$, $B, C)$.

Case $3-0<r\left(B_{1}\right)<m$ - The recovery conaition can be ordered as:

$$
\mathrm{V}_{2}\left(\mathrm{~B}_{1}+\mathrm{A}_{12} \Phi_{22} \mathrm{~B}_{2}\right)=\left(I+\mathrm{V}_{2} \mathrm{~A}_{12} \Phi_{22}\right) \mathrm{B}_{2}
$$

Now let $B_{1}$ be given as:

$$
B_{1}=T\left[\begin{array}{ll}
\Lambda & 0 \\
0 & 0
\end{array}\right] T^{-1}
$$

where $\Lambda$ is a diagonal matrix. The new transformed minimal-order observer parameters are:

$$
\begin{aligned}
& B_{1}^{\prime}=\left[\begin{array}{ll}
\Lambda & 0 \\
0 & 0
\end{array}\right], A_{11}^{\prime}=T^{-1} A_{11} T^{T} \\
& A_{21}^{\prime}=A_{21} T, A_{22}^{\prime}=A_{22}, V_{2}^{\prime}=V_{2} T \\
& A_{12}^{\prime}=T^{-1} A_{12}, E^{\prime}=E T, B_{1}^{\prime}=B_{2} T \\
& F^{\prime}=T^{-1} F, G^{\prime}=G T
\end{aligned}
$$

In the following the prime is suppressed, but the system is assumed to be in the transformed form.

$v_{2}$ can be written as:

$$
v_{2}=\underset{\left[\begin{array}{ll}
v_{21} & v_{22}
\end{array}\right]}{\stackrel{r\left(B_{1}\right)}{m-r\left(B_{1}\right)}}
$$

If this is inserted into $(A-7)$ it follows that:

$$
\begin{aligned}
& {\left[v_{21} \Lambda+v_{21}{ }^{A} 121_{22}{ }^{B_{21}}+v_{22^{A}}{ }_{122^{\Phi}} 22^{B_{21}}{ }^{\prime}\right.} \\
& \left.\mathrm{V}_{21}{ }^{\mathrm{A}} 121^{\Phi}{ }_{22} \mathrm{~B}_{22}+\mathrm{V}_{22}{ }^{\mathrm{A}} 122^{\Phi}{ }_{22}{ }^{\mathrm{B}_{22}}\right] \\
& =\left[B_{21}+V_{21} A_{121}{ }^{\Phi} 22^{B_{2}}{ }+V_{22^{A}}{ }_{122^{\Phi}} 22^{B_{21}}{ }^{\prime}\right. \\
& \left.v_{21}{ }^{A} 121^{\Phi} 22^{B_{22}}+B_{22}+v_{22}{ }^{A} 122^{\Phi} 22^{B_{22}}\right]
\end{aligned}
$$

$(A-11)$

Where $A_{12}$ and $B_{2}$ are partitioned compatibly with $\nabla_{2}$. Eq. (A-11) implies that:

$$
v_{21}=B_{21} \Lambda^{-1}
$$

\begin{tabular}{|c|c|c|c|}
\hline Observer-type & Diagram & Condition & Subject to \\
\hline Full-order & & $\frac{F}{\bar{q}} \rightarrow B x$ & $S(A, B, C)$ \\
\hline $\begin{array}{c}\text { Minimal-order } \\
x\left(B_{1}\right)=0\end{array}$ & & $\frac{v_{2}}{q} \rightarrow B_{2} \alpha$ & $\begin{array}{l}S\left(A_{22}, B_{2},\right. \\
\left.A_{12}\right)\end{array}$ \\
\hline $\begin{array}{l}\text { Minimal-order } \\
0<r\left(B_{1}\right)<m\end{array}$ & & $\frac{v_{2 q 2}}{q} \rightarrow B_{22}$ & $\begin{array}{l}S\left(\bar{A}_{22}, B_{22}\right. \\
\left.A_{122}\right)^{-2}\end{array}$ \\
\hline
\end{tabular}

$$
\mathrm{V}_{22}\left(\mathrm{I}+\mathrm{A}_{122} \Phi_{22} \mathrm{~V}_{22}\right)^{-1}=\mathrm{B}_{22}\left(\mathrm{~A}_{122^{\Phi}}{ }_{22} \mathrm{~B}_{22}\right)^{-1}
$$

Here $v_{2 I}$ is uniquely given, and the condition on $v_{22}$ is equivalent to the full-state LTR condition, hence:

$$
\begin{aligned}
& v_{2}=\left[\begin{array}{ll}
v_{20} & v_{2 q}
\end{array}\right], v_{20}=B_{2 \Lambda}{ }^{-1} \\
& \frac{v_{2 q}}{q} \rightarrow B_{22^{\alpha}}, q \rightarrow \infty, \operatorname{det}(\alpha) \neq 0 .
\end{aligned}
$$

With this selection of $\mathrm{v}_{2}$ the $\mathrm{p}$ finite roots of $A_{22}-V_{2} A_{12}$ are equal to the zeros of $S(A, B, C)$, and the remaining $n-m-p$ eigenvalues approach infinity. [s3].

A detailed exposition of this analysis is given in
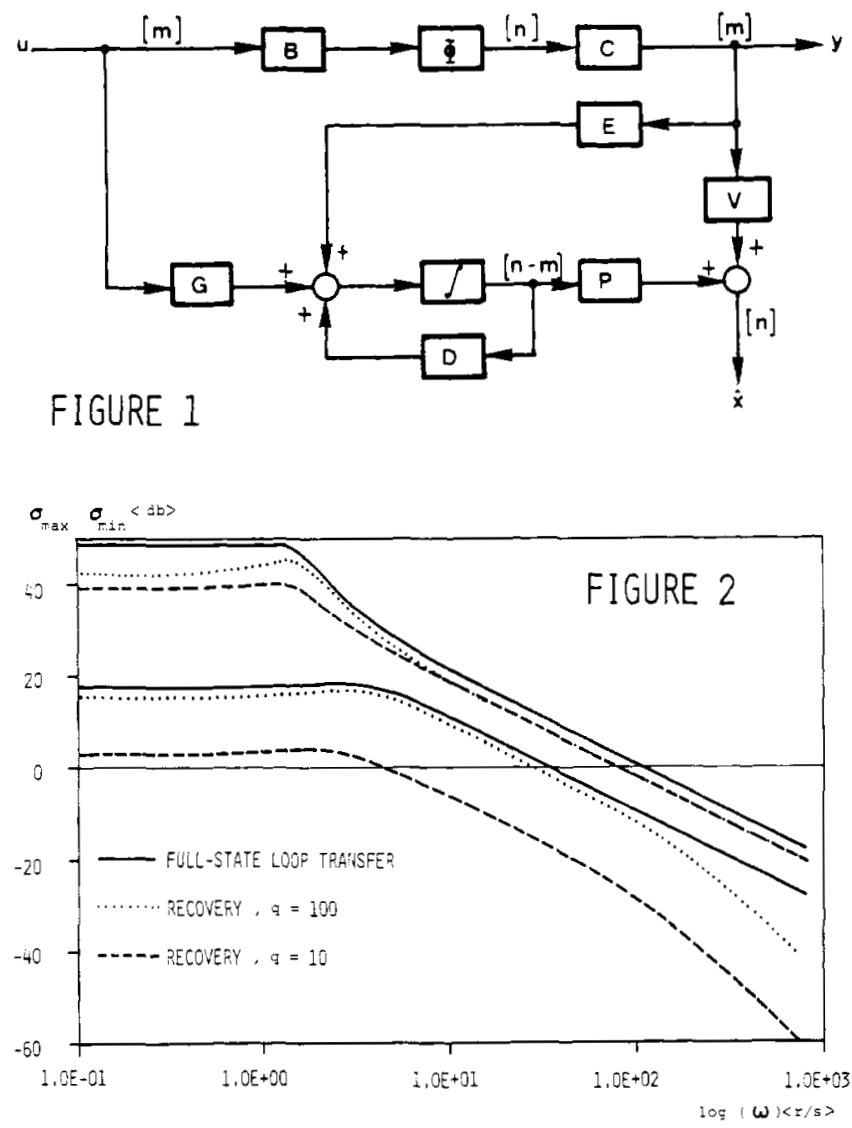

TABLE 1 